Supporting information for:

\title{
Influence of the Fluorination of Iron Phthalocyanine on the Electronic Structure of the Central Metal Atom
}

Katharina Greulich, ${ }^{\dagger}$ Martin Trautmann, ${ }^{\perp}$ Axel Belser, ${ }^{\dagger}$ Sven Bölke, ${ }^{\dagger}$ Reimer Karstens, ${ }^{\dagger}$ Peter Nagel, ${ }^{\#}$ Stefan Schuppler, ${ }^{\#}$ Michael Merz, ${ }^{\#}$ Angelika Chassé, ${ }^{\perp}$ Thomas Chassé, ${ }^{\dagger}{ }^{\ddagger}$ and Heiko Peisert ${ }^{\dagger *}$

${ }^{+}$Institute of Physical and Theoretical Chemistry, University of Tübingen, Auf der Morgenstelle 18, 72076

Tübingen, Germany

\# Karlsruhe Institute of Technology (KIT), Institute for Solid State Physics, 76021 Karlsruhe, Germany

$\perp$ Institute of Physics, Martin Luther University Halle-Wittenberg, Von-Danckelmann-Platz 3, 06120 Halle, Germany

* Center for Light-Matter Interaction, Sensors \& Analytics (LISA ${ }^{+}$) at the University of Tübingen, Auf der Morgenstelle 18, 72076 Tübingen, Germany

* Corresponding author, heiko.peisert@uni-tuebingen.de, Tel.: (+49) 07071 / 29-76931, Fax: (+49) 07071 / 29-5490

\section{Content:}

- Fe $2 p_{3 / 2}$ core level spectra of FePc and $\mathrm{FePcF}_{16}$ thin films on GeS

- XMCD signals of FePc and FePcF $F_{16}$ in dependence of magnetic field strength

- Calculation of the magnetic moments according to the sum rules

- Two parameter plots of FePcF ${ }_{16}$ for normal and grazing incidence with the calculation parameters $\mathrm{Dq}$ and Dt 
1. Fe $2 p_{3 / 2}$ core level spectra of FePc and FePcF 16 thin films on GeS
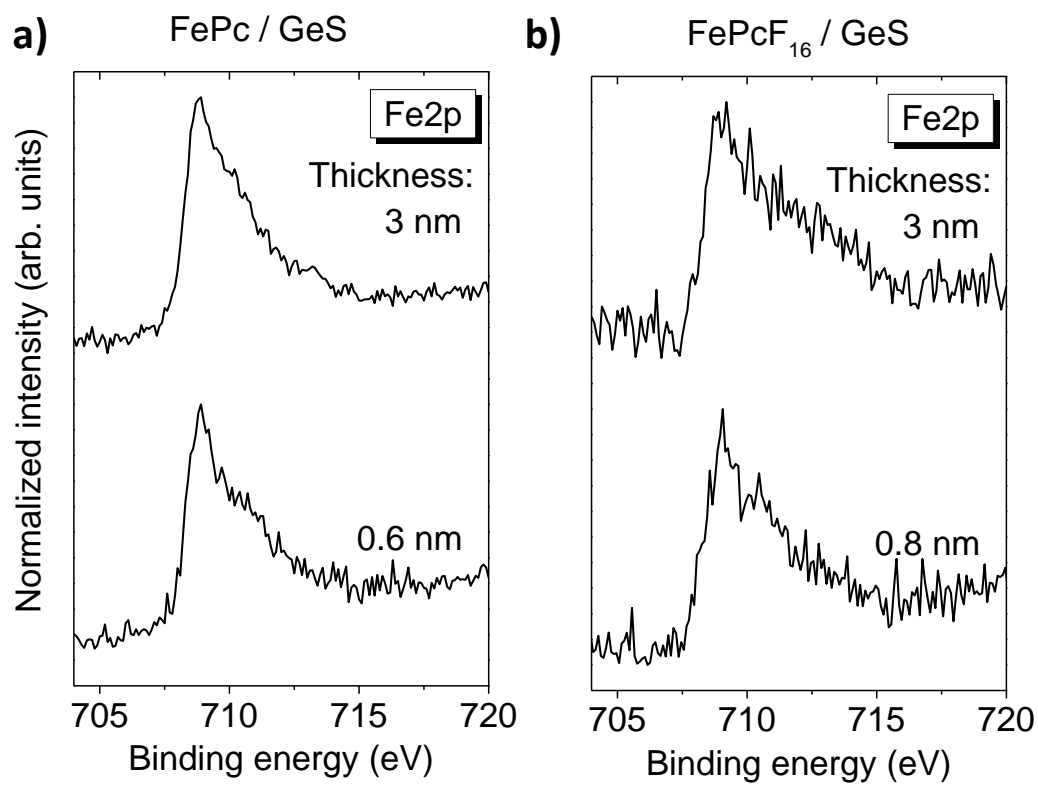

Figure S1. Fe $2 p_{3 / 2}$ core level spectra of FePc and $\mathrm{FePCF}_{16}$ on GeS as a function of film thickness. Both FePc and $\mathrm{FePCF}_{16} \mathrm{Fe} 2 \mathrm{p}$ core level spectra have the same shape, but the FePcF $\mathrm{F}_{16} \mathrm{Fe} 2 p$ peak looks slightly broader. There are no major changes in the spectral shape with increasing film thickness.

\section{2. $\mathrm{XMCD}$ signals of FePc and $\mathrm{FePcF}_{16}$ in dependence of magnetic field strength}

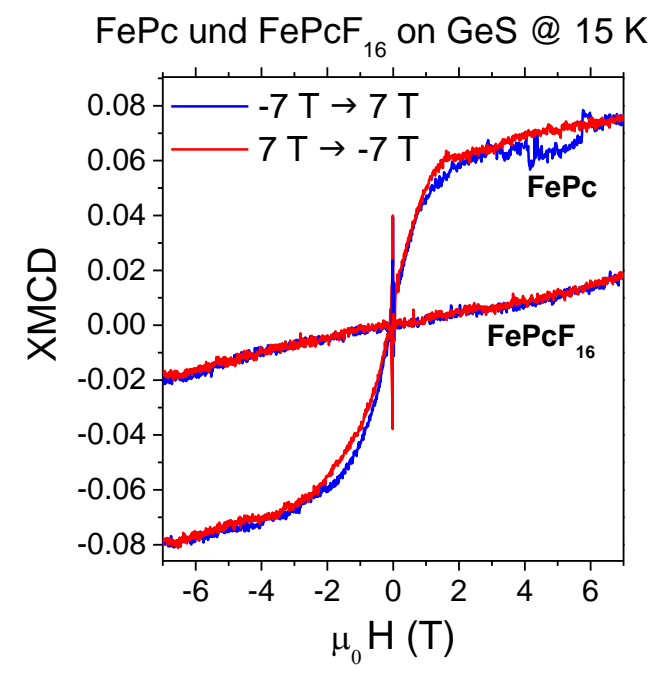

Figure S2. XMCD intensity, monitored at the photon energy of the most intense $X M C D$ signal at the Fe $L_{3}$ edge ( $h v=707.5 \mathrm{eV}$ for FePc and $h v=710.15 \mathrm{eV}$ for FePcF $F_{16}$ ). The blue curves are measured while ramping from $-7 T$ to $+7 T$, the red curves are measured in the opposite direction. For the small XMCD signal of $\mathrm{FePcF}_{16}$, we see a small, linear increase with magnetic field strength. The FePc curves show a sigmoidal behavior, but no open loop. 


\section{Calculation of the magnetic moments according to the XMCD sum rules}

Estimates for the orbital and spin components of the magnetic moments can be derived using the XMCD sum rules: $:^{1-6}$

$$
\begin{gathered}
\frac{m_{l}}{\mu_{B}}=-\frac{4}{3} n_{h} \frac{\int_{L 2+L 3} d \omega\left(\mu^{+}-\mu^{-}\right)}{\int_{L 2+L 3} d \omega\left(\mu^{+}+\mu^{-}\right)} \\
\frac{m_{S}^{e f f}}{\mu_{B}}=-n_{h} \frac{6 \int_{L 3} d \omega\left(\mu_{+}-\mu_{-}\right)-4 \int_{L 3+L 2} d \omega\left(\mu_{+}-\mu_{-}\right)}{\int_{L 3+L 2} d \omega\left(\mu_{+}+\mu_{-}\right)}
\end{gathered}
$$

where $m_{l}$ is the angular-dependent orbital component of the magnetic moment, $m_{s}^{e f f}$ is the effective spin component, and $n_{h}$ is the number of $\mathrm{d}$-holes above the Fermi energy. The integration ranges are 705$726 \mathrm{eV}$ for $L 2+L 3$ and $705-715 \mathrm{eV}$ for $L 3$. As an approximation for the unpolarized x-ray absorption white line intensity in the denominator, the integral of $\left(\mu_{+}+\mu_{-}\right)$has been used. Before integration, a function with steps at the $L_{2}$ and $L_{3}$ edges has been substracted from the spectrum to get rid of intensity from transitions into the continuum. The moments $m_{l}$ and $m_{s}^{e f f}$ calculated from the XMCD spectra of FePc and FePcF $_{16}$ on GeS are shown in Table S1 The values for FePc are in good agreement with literature. ${ }^{7}$

Table S1. Magnetic Moments $m_{l}$ and $m_{s}^{\text {eff }}$ as determined from the XMCD spectra (7T, $\left.15 \mathrm{~K}\right)$ of $3 \mathrm{~nm} \mathrm{FePc}$ and $\mathrm{FePCF}_{16}$ on GeS.

\begin{tabular}{lcc}
\hline & $\boldsymbol{m}_{\boldsymbol{l}} / \boldsymbol{n}_{\boldsymbol{h}} / \boldsymbol{\mu}_{\boldsymbol{B}}$ & $\boldsymbol{m}_{\boldsymbol{s}}^{\boldsymbol{e f f}} / \boldsymbol{n}_{\boldsymbol{h}} / \boldsymbol{\mu}_{\boldsymbol{B}}$ \\
\hline FePc grazing incidence & $0.17( \pm 0.02)$ & $0.22( \pm 0.03)$ \\
FePc normal incidence & $0.10( \pm 0.004)$ & $-0.02( \pm 0.01)$ \\
FePcF $_{16}$ grazing incidence & $0.02( \pm 0.01)$ & $0.03( \pm 0.02)$ \\
FePcF $_{16}$ normal incidence & $0.03( \pm 0.005)$ & $-0.03( \pm 0.008)$ \\
\hline
\end{tabular}

Under the assumption of flat lying molecules with a random orientation in the $x y$-plane, the angulardependent orbital moment can be split into components parallel $\left(m_{l}^{x y}\right)$ and normal $\left(m_{l}^{z}\right)$ to the substrate plane:

$$
m_{L}(\theta)=m_{l}^{z} \sin ^{2}(\theta)+m_{l}^{x y} \cos ^{2}(\theta) .
$$

In addition, the effective spin moment $m_{s}^{\text {eff }}$ is composed of the spin moment $m_{s}$ and the angulardependent magnetic dipole moment $m_{t}$

$$
m_{s}^{e f f}(\theta)=m_{s}-7\left(m_{t}^{z} \sin ^{2}(\theta)+m_{t}^{x y} \cos ^{2}(\theta)\right) .
$$


For $3 \mathrm{~d}$ electron systems, the angular average of $m_{t}$ vanishes and one can write: ${ }^{8}$

$$
m_{t}^{z}+2 m_{t}^{x y}=0,
$$

which makes the determination of $m_{s}$ and $m_{t}$ with measurements at only two angles possible (c. $f$. Table S2).

Table S2. Orbital moments $m_{l}^{z}, m_{l}^{x y}$ and spin moment $m_{s}$, together with the magnetic dipole moment $m_{t}^{z}$, as derived from the XMCD sum rule results of the FePc and FePcF ${ }_{16}$ thin films on GeS.

\begin{tabular}{lccccc}
\hline & $m_{l}^{z} / n_{h} / \mu_{B}$ & $m_{l}^{x y} / n_{h} / \mu_{B}$ & $m_{s} / n_{h} / \mu_{B}$ & $m_{t}^{z} / n_{h} / \mu_{B}$ & $\frac{m_{l}^{x y}}{m_{s}}$ \\
\hline FePc & $0.10( \pm 0.004)$ & $0.19( \pm 0.03)$ & $0.19( \pm 0.03)$ & $0.03( \pm 0.004)$ & 1 \\
FePcF 16 & $0.03( \pm 0.005)$ & $0.02( \pm 0.005)$ & $0.02( \pm 0.01)$ & $0.01( \pm 0.002)$ & 1 \\
\hline
\end{tabular}


4. Two parameter plots of FePcF 16 for normal and grazing incidence with the calculation parameters $\mathrm{Dq}$ and $\mathrm{Dt}$

(a) Intensity XMCD
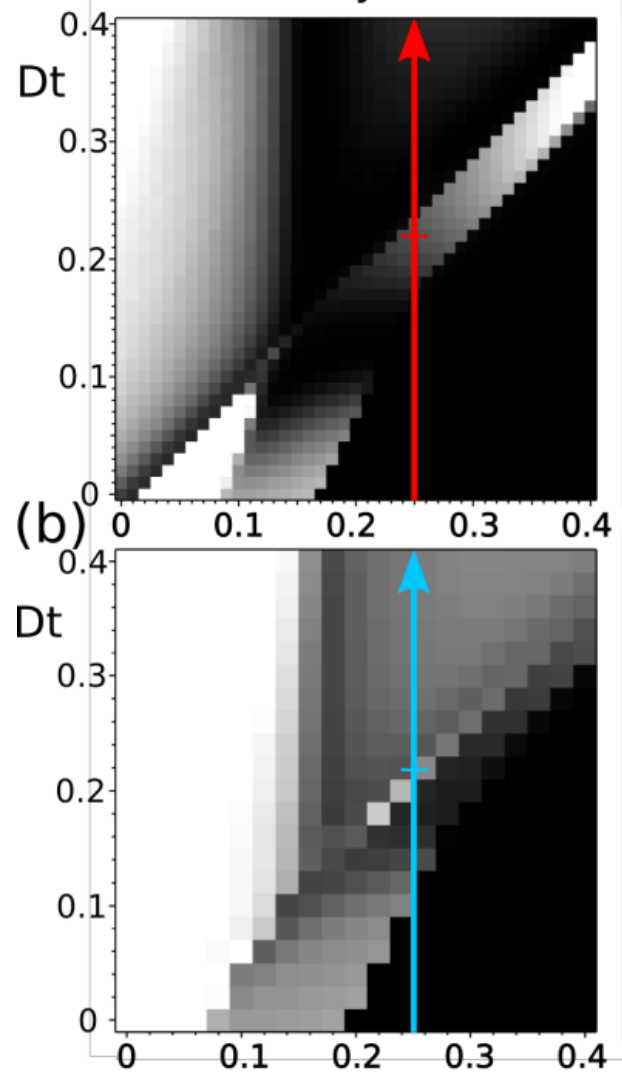

(c)

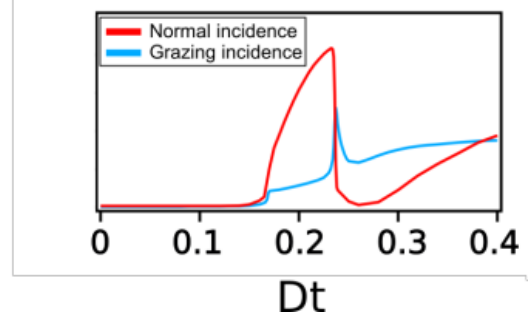

$m_{1}$
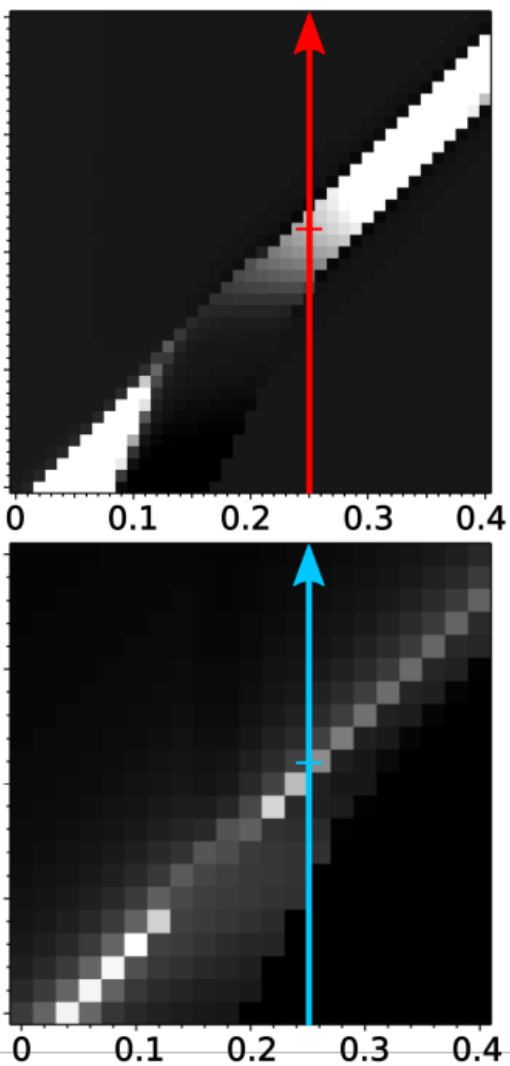

$\mathrm{Dq}$

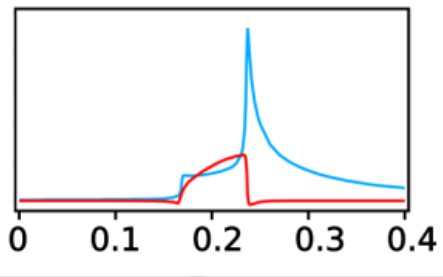

Dt $m_{s}{ }^{\text {eff }}$
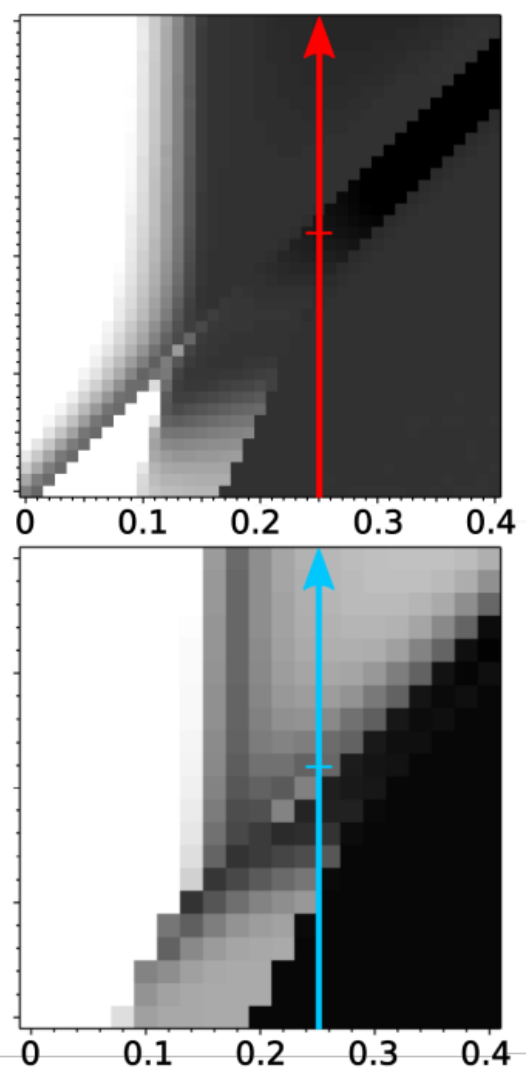

$\mathrm{Dq}$

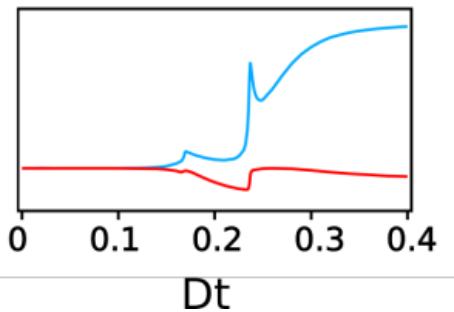

Figure S3. Two parameter plots of FePcF $\mathrm{F}_{16}$ for (a) normal and (b) grazing incidence with the calculation parameters $D q$ and $D t$. In the greyscale black corresponds to low and white to high values for XMCD, $m_{l}$ and $m_{s}^{e f f}$ respectively. The line profiles in (c) are taken along the red and blue arrows. The small crosses along the arrows mark the calculation parameter used for FePcF $F_{16}$. 


\section{References}

1. Thole, B. T.; Carra, P.; Sette, F.; Van der Laan, G., X-Ray Circular-Dichroism as a Probe of Orbital Magnetization, Phys. Rev. Lett. 1992, 68, 1943-1946.

2. Carra, P.; Thole, B. T.; Altarelli, M.; Wang, X. D., X-Ray Circular-Dichroism and Local Magnetic-Fields, Phys. Rev. Lett. 1993, 70, 694-697.

3. Altarelli, M., Orbital-Magnetization Sum Rule for X-Ray Circular Dichroism: A Simple Proof, Phys. Rev. B 1993, 47, 597-598.

4. Stöhr, J.; König, H., Determination of Spin- and Orbital-Moment Anisotropies in Transition Metals by Angle-Dependent X-Ray Magnetic Circular Dichroism, Phys. Rev. Lett. 1995, 75, 3748-3751.

5. Weller, D.; Stöhr, J.; Nakajima, R.; Carl, A.; Samant, M. G.; Chappert, C.; Mégy, R.; Beauvillain, P.; Veillet, P.; Held, G. A., Microscopic Origin of Magnetic Anisotropy in Au/Co/Au Probed with X-Ray Magnetic Circular Dichroism, Phys. Rev. Lett. 1995, 75, 3752-3755.

6. Chen, C. T.; Idzerda, Y. U.; Lin, H. J.; Smith, N. V.; Meigs, G.; Chaban, E.; Ho, G. H.; Pellegrin, E.; Sette, F., Experimental Confirmation of the X-Ray Magnetic Circular Dichroism Sum Rules for Iron and Cobalt, Phys. Rev. Lett. 1995, 75, 152-155.

7. Bartolomé, J.; Bartolomé, F.; Garcia, L. M.; Filoti, G.; Gredig, T.; Colesniuc, C. N.; Schuller, I. K.; Cezar, J. C., Highly Unquenched Orbital Moment in Textured Fe-Phthalocyanine Thin Films, Phys. Rev. B 2010, 81.

8. Merz, M.; Nagel, P.; Pinta, C.; Samartsev, A.; v. Löhneysen, H.; Wissinger, M.; Uebe, S.; Assmann, A.; Fuchs, D.; Schuppler, S., X-Ray Absorption and Magnetic Circular Dichroism of $\mathrm{LaCoO}_{3}, \mathrm{La}_{0.7} \mathrm{Ce}_{0.3} \mathrm{CoO}_{3}$, and $\mathrm{La}_{0.7} \mathrm{Sr}_{0.3} \mathrm{CoO}_{3}$ Films: Evidence for Cobalt-Valence-Dependent Magnetism, Phys. Rev. B 2010, 82, 174416. 